\title{
Acute kidney injury, a common and severe complication in hospitalized patients during the COVID-19 pandemic
}

\author{
Roberto Scarpioni ${ }^{1} \cdot$ Teresa Valsania $^{1}$ - Vittorio Albertazzi ${ }^{1}$ - Valentina Blanco ${ }^{1}$ - Sara DeAmicis ${ }^{1}$ - Alessandra Manini ${ }^{1}$. \\ Luigi Melfa ${ }^{1} \cdot$ Marco Ricardi $^{1}$. Chiara Rocca ${ }^{1}$. Stefano Gandolfi ${ }^{2}$
}

Received: 27 December 2020 / Accepted: 1 April 2021 / Published online: 19 June 2021

(c) Italian Society of Nephrology 2021

\begin{abstract}
Introduction Acute respiratory failure (ARF) is the main clinical sign of coronavirus disease-2019 (COVID-19), but little is known about the outcome of acute kidney injury (AKI) associated with ARF.

Study design Retrospective cohort study on clinical features of adult patients hospitalized with COVID-19 between March 1st and April 30th, 2020 in the district of Piacenza (Italy).

Results Among 1894 hospitalized patients, 1701 affected by COVID-19 underwent at least two serum creatinine evaluations. According to KDIGO definitions, 233 of 1,701 patients (13.7\%) developed AKI: 159, 34, and 40 had stage 1, 2 and 3 AKI, respectively. Patients with AKI were older (mean age 73.5 \pm 14 years, range 24-95) than those without AKI (72 \pm 14 years, range 20-102). In-hospital mortality was high in COVID patients (567/1701 patients, 33\%), which almost doubled among AKI patients $(132 / 233$ patients, $57 \%)$, compared with those without AKI $(p<0.01)$. Risk factors for AKI included older age, male gender, diabetes and need for ventilation. Fourteen patients with stage 3 AKI underwent renal replacement therapy (RRT). Conclusions Hospitalized COVID-19 patients with AKI associated with ARF have poor chances of survival. Diagnosing and preventing the progression of renal damage is fundamental in order to delay initiating RRT, especially when resources are limited.
\end{abstract}

Keywords Acute kidney injury $\cdot$ COVID-19 $\cdot$ AKI severity $\cdot$ Mortality $\cdot$ Renal replacement therapy $\cdot$ Dialysis

\section{Introduction}

On December 31st, 2019, an outbreak of pneumonia of unknown etiology was reported in Wuhan (China) and the cause of this infection was identified by Chinese researchers (Chinese CDC) as a new coronavirus called SARS-CoV-2 or COVID-19 [1]. One month later, on January 31st, two Chinese tourists from the city of Wuhan on vacation in Rome were the first COVID-19 patients to be identified and treated in Italy.

On February 21st, 2020, the first Italian patient with COVID-19 was admitted to Codogno Hospital (Lodi) just $15 \mathrm{~km}$ from Piacenza [2]. Over the next two weeks there followed an exponential growth in the number of COVID-19

Roberto Scarpioni

r.scarpioni@ausl.pc.it

1 Department of Nephrology and Dialysis, ASL "Guglielmo da Saliceto" Hospital Piacenza, via Taverna 49,

29121 Piacenza, Italy

2 Department of Staff, ASL “Guglielmo da Saliceto" Hospital, Piacenza, Italy infections: soon Italy was one of the hardest hit countries worldwide, with 1,651,230 confirmed cases and more than 100,000 deaths. The Emilia-Romagna region (where Piacenza is located), reported 276,604 cases, among whom 10,746 died and 213,413 were declared cured [3]. The pandemic quickly spread to all continents with $115,653,459$ confirmed cases and 2,571,823 deaths [4]. Patients admitted to hospital with COVID-19 were critically ill with acute respiratory distress syndrome (ARDS) often leading to the need for mechanical ventilation: but what about renal involvement?

The incidence of acute kidney injury (AKI) during the first wave of the on-going COVID-19 pandemic was recently investigated by several authors [5-11]. More than 15 years ago, Chu et al. [12] described AKI in ARDS related to a coronavirus: a relatively small number (36 of 536 patients, 6.7\%) developed AKI, however a dramatic number, 33 of $36(91.7 \%)$ died. Due to the strong link between SARS-CoV and COVID-19, extrapolation of the results led to the assumption that AKI was uncommon in SARS, but carried a high mortality [12]. During the COVID-19 pandemic that has spread all over the world, some reports found the rate of AKI ranging from 
0.5 to $46 \%[9,13-17]$ with an incidence rate of patients requiring dialysis varying from 14 to $19 \%$ of those with AKI.

\section{Patients and metods}

\section{Study design and cohort}

Here we report a retrospective observational cohort study carried out in four hospitals in the Piacenza district (northwestern Italy), one of the hardest hit areas by the COVID-19 pandemic in Italy, and studied various baseline characteristics as potential predictors of AKI in COVID-19.

Data sources: the demographic characteristics and laboratory data extracted from the electronic medical records of the Clinical Laboratory, ASL “Guglielmo da Saliceto" Hospital in Piacenza were analyzed. All consecutive patients older than 18 years of age, admitted to our hospitals from March 1st to April 30th, 2020 who were positive for COVID-19 by polymerase chain reaction testing of a nasopharyngeal sample were eligible. In case of multiple hospital admissions, only the first one was considered. See flowchart.
The date of AKI onset was defined as the earliest day of a change in serum creatinine meeting the Kidney Disease Improving Global Outcomes (KDIGO) criteria. AKI stage was determined using the peak serum creatinine level after AKI detection. AKI was defined using the KDIGO criteria [15], and severity of AKI was rated as follows: stage $1=$ increase in serum creatinine by $0.3 \mathrm{mg}$ / $\mathrm{dL}$ within $48 \mathrm{~h}$ or a 1.5-1.9 times increase in serum creatinine from baseline within 7 days; stage $2=2-2.9$ times increase; stage $3=3$ times or more increase in serum creatinine within 7 days or initiation of renal replacement therapy (RRT). The upper limits of normal serum creatinine considered in men and women were 1.2 and $1 \mathrm{mg} /$ $\mathrm{dL}$, respectively.

Patients were stratified according to the highest AKI stage attained during their hospital stay. Baseline serum creatinine level was defined as the value at hospital admission. Baseline characteristics as potential risks for AKI in COVID-19 patients were analyzed (see Table 1, Baseline characteristics of patients affected by COVID-19 at
Hospital adult admissions with positive Covid-19 tests

between March $1^{\text {st }}$ and April 31 ${ }^{\text {th }}, 2020$ (data from 4

Hospitals in Piacenza district)

$n=1,894$ admissions of

1,798 patients

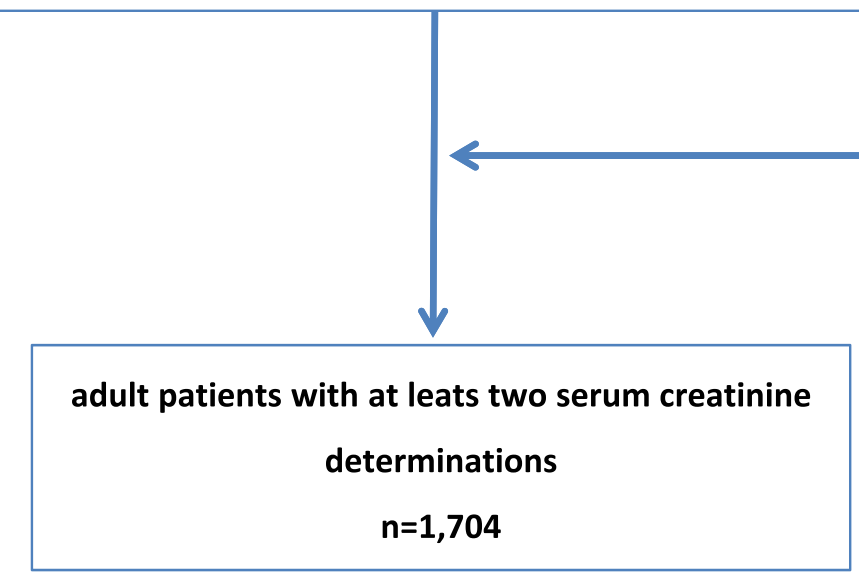

Excluded:

- Age $<18$ years

- Kidney transplant $(n=4$, one of whom admitted to ICU at Bologna Hospital for respiratory failure)

- $\operatorname{ESKD~(~} n=49,21$ of whom died)

- Serum creatinine not available or only one determination available $n=35$

- Self discharge from Hospital against medical opinion $n=6$

- Transferred to hospitals out of Piacenza district

*In case of multiple admissions for one patient only the first episode was included $n=96$ 
Table 1 Baseline characteristics of patients affected by COVID19 at hospital admission

\begin{tabular}{lrrrlllc}
\hline & $n^{\circ}$ & $\begin{array}{l}\text { mean } \\
\text { age } \pm \text { SD }\end{array}$ & $\begin{array}{l}\text { Dia- } \\
\text { betes } \\
(\mathrm{n})\end{array}$ & $\begin{array}{l}\text { Deceased } \\
\left(n^{\circ}\right)\end{array}$ & Gender $(\mathrm{M} / \mathrm{F})$ & $\begin{array}{l}\text { Patients on } \\
\text { ventilator }\end{array}$ & $\begin{array}{l}\text { Invasive } \\
\text { mechanical } \\
\text { ventilation }\end{array}$ \\
\hline AKI* $^{*}$ & 233 & $73.5 \pm 11.9$ & 49 & $132(57 \%)$ & $171 / 62$ & 37 & 69 \\
NO AKI* & 1468 & $71.8 \pm 14.0$ & 180 & $435(30 \%)$ & $922 / 546$ & 169 & 171 \\
Total & 1701 & & 229 & 567 & $1093 \mathrm{M} / 608 \mathrm{~F}$ & 206 & 240 \\
\hline
\end{tabular}

*With at least two different determinations of serum creatinine hospital admission). The incidence of AKI and short-term outcomes were also evaluated. Baseline characteristics as potential risks for AKI in COVID-19 patients were analyzed (see Table 2, potential independent risks of AKI in COVID-19 by multivariate analysis).

Patients affected by end stage kidney disease (ESKD), kidney transplant recipients, and subjects without two consecutive serum creatinine determinations were excluded from the analysis. The local Ethics Committee of the ASL "Guglielmo da Saliceto" Hospital in Piacenza approved the study (authorization obtained on August 5th, 2020, reference number: $780 / 2020 /$ OSS $^{*}$ ). The study was carried out in accordance with the Helsinki Declaration.

Written informed consent was obtained from all patients at hospital admission.

\section{Statistical analysis}

We compared baseline characteristics among patients with or without AKI using the chi-square test for categorical variables. Continuous data matching normal distribution mean \pm standard deviation, continuous data not matching normal distribution median and min-max, were given. Logistic regression analysis was used to detect independent predictors of the development of AKI. Data were analyzed using STATA 9 for Windows; $p<0.05$ was considered significant in all tests.

\section{Data cleaning}

The data were reviewed by a trained team of physicians. To ensure accurate exclusion of ESKD and kidney transplant a comparative analysis between two data records was carried out (ASL Hospital Piacenza and electronic registry of the Nephrology and Dialysis Center, ASL Piacenza).

\section{Results}

Of 1701 patients with COVID-19 admitted to Piacenza District Hospitals from March 1st to April 30th, 2020, AKI developed in 233 patients (13.7\%). The peak stages of AKI were stage 1 in $65 \%(n=153)$, stage 2 in $15 \%(n=34)$, and stage 3 in $17 \%(n=40)$; of the latter group, 14 patients $(6 \%$ of all patients developing AKI) required RRT. Hospitalized patients who developed AKI were older $(73.5 \pm 11.9$ years $)$ compared with no-AKI $(71.8 \pm 14)$ and more than half of the patients with AKI had a significantly increased risk of death (132 of 233 patients) compared with no-AKI (435 of 1468), $p<0.01$. There was an increasing risk of death with increasing stages of AKI, with the highest risk seen in patients with AKI stage 3: among deceased patients, 81 of 132 (61\%) had AKI stage 1, 21 had stage $2(16 \%)$, and $30(23 \%)$ had stage 3. Diabetes was present in 233 of 1701 hospitalized patients (14\%), a prevalence that is higher than the overall incidence of diabetes in the Emilia-Romagna region (about 7\%). Among 1701 hospitalized patients, 49 with diabetes developed AKI. ARDS was the most worrisome clinical condition in 446 of 1,701 admitted patients (26\%), in 340 with no-AKI and in 106 with AKI: among the latter, 37 were on a ventilator and 69 were on mechanical ventilation, confirming the predominant feature of respiratory failure among hospitalized patients with COVID-19 infection.
Table 2 Potential independent risks of AKI in COVID-19 by multivariate analysis

\begin{tabular}{llllll}
\hline AKI & $\begin{array}{l}\text { Unadjusted Odds } \\
\text { ratio }\end{array}$ & $\begin{array}{l}\text { Standard } \\
\text { error }\end{array}$ & $\mathrm{z}$ & $\begin{array}{l}p>z \\
\text { value }\end{array}$ & $\begin{array}{l}\text { [95\% confidence } \\
\text { interval] }\end{array}$ \\
\hline Age & 1.03 & 0.0067729 & 4.5 & 0 & $1.02-1.04$ \\
Male gender & 1.53 & 0.2544119 & 2.57 & 0.01 & $1.11-2.12$ \\
Patients on ventilator & 2.07 & 0.4364701 & 3.45 & 0.001 & $1.37-3.13$ \\
Natremia & 1.00 & 0.0015658 & 1.55 & 0.121 & $1.0-1.01$ \\
$\begin{array}{l}\text { Patients on invasive mechanical } \\
\text { ventilation }\end{array}$ & 4.73 & 0.9072937 & 8.09 & 0 & $3.24-6.88$ \\
\hline
\end{tabular}


Among 233 patients who developed AKI during hospitalization, a total of 132 died, while 20 were discharged or transferred to another hospital or facility due to the lack of beds in all district hospitals. Eleven of the 14 patients who required continuous renal replacement therapy (CRRT) were males. Nine patients died and 5 were discharged from the hospital, only two of whom had no need for chronic dialysis. All patients requiring CRRT had co-morbidities (10 had hypertension, 6 had a baseline mild stage of CKD, 6 had a history of coronary artery disease). Altogether, 89 CRRT treatments were administered over two months, with a mean of 7 dialysis sessions per patient, range 2-31. In the hardest, most overcrowded days of the pandemic, due to the absolute lack of ICU beds, 157 patients were transferred to other hospitals outside the District of Piacenza, and among them, 20 developed AKI. CRRT was needed for 3 patients; one of them eventually recovered renal function, while the other two are still on dialysis. Noteworthy, of the 101 hospitalized patients who survived after developing AKI, as many as 20 $(=20 \%)$ died at home after being discharged. Multivariate analysis revealed that increased age, male gender, diabetes and the need for mechanical ventilation were independent risks predictors of AKI in COVID-19.

\section{Discussion}

The exact mechanism of AKI due to COVID-19 is unclear. AKI occurring during COVID-19 infection may depend on complex and multifactorial causes, as described in Table 3, and an important role seems to be related to angiotensinconverting enzyme (ACE) 2 protein. See Table 3 causes of renal damage.

The main binding site for SARS-CoV-2 is the ACE2 protein, which is expressed in the kidney much more than in the lungs [18-20]. ACE2 is expressed on the brush border apical membrane of the proximal tubule, where it co-localizes with ACE, and it is also present at lower levels in podocytes [19]. It is conceivable that the virus could enter the kidney by invading the podocytes first, and thus the tubular fluid, and subsequently bind to ACE2 in the proximal tubule: confirming this, Su et al. [21] observed the presence of virus-like particles in podocytes and renal tubular epithelial cells by electron microscopy. Recent clinical and autopsy reports of COVID-19 from China and the United States confirm increased clotting and disseminated intravascular coagulation with small vessel thrombosis and pulmonary, splenic and renal infarction [22]. Further, elevated D-dimer and low platelet levels correlated with worse outcomes [23].

In the aforementioned study by Hirsh et al. [14] from New York (USA), 66\% of the patients with AKI had a urine Na of $0.35 \mathrm{mmol} / \mathrm{L}$, suggestive of hypovolemia, a prerenal state, or low effective arterial blood volume from heart failure: in our analysis natremia was not found to be an independent risk of AKI (see Table 1). Despite the very limited availability of information on kidney involvement in COVID-19, AKI appears to include a complex process driven by virusmediated injury, cytokine storm, ANG2 pathway activation, dysregulation of complement, hypercoagulation, and microangiopathy interacting with common and known risk factors for AKI.

AKI has been reported among patients with COVID-19 infection with wide-ranging incidence rates, i.e., from 0.5 to $46 \%$ in hospitalized patients $[9,13,17]$, to $19 \%$ of subjects in the ICU setting [24], and as high as $90 \%$ in patients requiring mechanical ventilation [13].

A similar incident rate was reported by a joint research survey carried out by the Italian Society of Nephrology (SIN) and the Italian Society of Anesthesiology, Resuscitation and Intensive Care (SIAARTI) (available at: http:// www.siaarti.it/News/surveycovid-19AKIRRT.aspx, accessed on $13^{\text {th }}$ September, 2020 at 23.00 h): among 19,000 hospitalized patients affected by COVID-19 (1393 of whom developed AKI [13\%]), 82\% were hospitalized in the ICU (1146 of 1393). Interestingly, about $25 \%$ of AKI occurrences were reported in patients with ARDS [6]. These data were recently confirmed by the Italian National Report of the "Istituto Superiore di Sanità" describing an incidence of 27.8\% among more than 2000 patients (https://www.epice ntro.iss.it/corona-virus/bollettino/Report-COVID-2019_17_ marzo-v2.pdf, updated on March 17th, 2020).

All these data differ from the experience reported by a Chinese group [25] which found AKI to be uncommon among 116 confirmed COVID-19 patients. SARS-CoV-2 infection did not result in AKI, nor did it worsen CKD in COVID-19 patients.

In renal epithelial cells, viral infection may worsen local inflammatory response and consequently, the incidence and duration of AKI episodes: previous CKD, when present,

Table 3 Causes of renal damage related to COVID-19

Hypotension and reduced kidney perfusion

Cytokine storm syndrome

Direct viral infection of the kidneys with virus-induced cytopathic injury through an ACE2 dependent pathway
Small vessel thrombosis Inflammation and sepsis Drug-induced nephrotoxicity Increased vascular permeability

ACE2 angiotensin-converting enzyme 2 
might therefore exacerbate renal damage. Thus, early identification of patients at risk of developing AKI might lead to early preventive interventions, thereby allowing better allocation of resources, which are likely to be especially limited during a pandemic.

In our experience, the incidence of AKI was less frequent (13.7\%) compared with 37\% reported by Hirsh et al. [14] in the New York area in a study involving 5,500 patients with COVID-19, or compared with $46 \%$ of 3993 patients analyzed by Chan et al. [26]. In our experience, in-hospital mortality was dramatically high (57\%), comparable to what was described at Mount Sinai Hospital, USA [25] which reported 50\% mortality in AKI versus $8 \%$ among those without AKI, significantly lower than our records among nonAKI patients with COVID-19 (30\%). The lower incidence of AKI in our study may reflect key differences in our cohort, including lower proportion of patients with co-morbidities, diabetes and chronic kidney diseases, all of which are risk factors for developing AKI.

One consideration regarding the lower incidence of AKI in our experience may be related to the reduced availability of ICU beds for the more complicated, often elderly patients with co-morbidities in the days of enormous influx to the hospital. These patients may actually have had renal failure, but the lack of ICU beds and the impossibility to ensure ventilation support resulted in reduced survival, which may have consequently prevented us from identifying the occurrence of AKI, as well as not allowing the patients enough time to develop AKI. In-hospital mortality percentages of patients with AKI in the ICU were markedly higher than those without AKI, as was the need for respiratory support.

All patients in the ICU received CRRT in separate rooms by veno-venous therapies because the severity of the clinical conditions of ARDS patients did not allow us to consider PD as a first-line technique for acute patients, unlike other experiences that considered an acute PD program as a viable option for acute patients during Covid infection [27].

However, considering the lack of specific treatment against COVID-19, we used sorbent cartridges designed to remove cytokines and other circulating inflammatory mediators (as is the strategy adopted in cases of septic shock) in an effort to counter the virus-triggered cytokine storm. Unfortunately, this may not have translated into improved patient outcome.

Our observational retrospective study, which is limited to the Piacenza area during the first peak of the COVID-19 pandemic, presents some limitations: first we calculated the baseline serum creatinine as the value on the day of hospital admission not knowing whether AKI was present at that moment. Furthermore, we did not evaluate patients with normal serum creatinine levels, which may have resulted in underestimating our data regarding the onset of AKI during hospitalization.
Second, as with other similar observational studies, we can only postulate about the relationship between viral exposure and AKI: renal biopsies were banned because of the high risk of spreading the virus during the maneuvers and the preparation of the histological material. Third, we based our observations on electronic health information alone, nonetheless, all data were revised and critically analyzed by all the authors. Four, since we only studied hospitalized patients we cannot assume that non-hospitalized patients might have had different renal involvement, thus albeit unlikely, it can not be ruled out. Fifth, there is a lack of urinanalysis information for all patients, which is not available through the database due to the impossibility to collect these data during such an intense period when all our resources are fully focused on providing clinical respiratory support to the patients. The dramatic and lightning-fast spread of the epidemic forced us to double the number of hospital beds in the Piacenza Hospital district from 350 to 700 so as to support all the patients in need of respiratory aid. Another limitation is the lack of data concerning approximately 157 patients who needed respiratory support because of ARDS, but who were hospitalized in other facilities due to an insufficient number of ICU beds some days, even though the amount of ICU beds rapidly increased from 16 to 50 .

However, the main strength of our single-center study is that it involves one of the largest cohorts of hospitalized patients with COVID-19 and AKI, and it has optimal data homogeneity.

\section{Conclusions}

At a time when the COVID-19 pandemic is still extremely widespread, our experience might guide other nephrologists to consider AKI as a relatively likely consequence of COVID-19 infection which can result in very high mortality rates, especially in CKD patients with other co-morbidities.

Awareness of kidney disease in patients with COVID-19 needs to be raised among clinicians: our findings suggest that AKI is a relatively frequent complication of COVID-19 as well as a marker of disease severity. Increased age, male gender, diabetes and the need for mechanical ventilation are all predictors of AKI.

The risk of dying in the hospital was significantly higher, almost twice as high, when AKI developed, and above all among those who needed dialysis support. It is imperative to identify AKI as soon as possible in order to prevent progression of kidney damage in an effort to delay initiation of RRT, especially during a pandemic if resources are limited. 
Author contributions RS and TV designed the study. MR, VA, SD, AM, CR, LM, SG and VB collected the data and prepared the figures and tables. RS and SG contributed analytical tools. RS, TV and SG wrote the paper. RS and SG conceived the project and supervised and coordinated all the work.

\section{Declarations}

Conflict of interest The authors declare no conflicts of interest.

Research involving human participants and/or animals The local Ethics Committee of the ASL "Guglielmo da Saliceto" Hospital in Piacenza approved the study (authorization obtained on August 5th, 2020, reference number: 780/2020/OSS*). The study was carried out in accordance with the Helsinki Declaration.

Informed consent Written informed consent was obtained from all patients at hospital admission.

\section{References}

1. Zhu N, Zhang D, Wang W et al (2020) A novel Coronavirus from patients with pneumonia in China, 2019. N Eng J Med 382(8):727-733. https://doi.org/10.1056/NEJMoa2001017)

2. Scarpioni R, Manini A, Valsania T et al (2020) COVID-19 and its impact on nephropathic patients: the experience at Ospedale "Guglielmo da Saliceto" in Piacenza. G Ital Nefrol 37(2):2020vol2 (PMID: 32281756)

3. Source: Dipartimento Protezione Civile, Italian National Government of Health (2020). http://www.salute.gov.it/portale/nuovo coronavirus/dettaglioContenutiNuovoCoronavirus.jsp?lingua $=$ italiano $\& \mathrm{id}=5338 \&$ are $=$ nuovoCoronavirus $\&$ menu $=$ vuoto. Accessed 6 Mar 2021

4. World Health Organization (2020) Health Emergency Dashboard, Available at Italian National Government of Health http://www. salute.gov.it/portale/nuovocoronavirus/dettaglioContenutiNu ovoCoronavirus.jsp?lingua $=$ italiano $\& \mathrm{id}=5338 \&$ are $a=$ nuovo Coronavirus\&menu=vuoto. Accessed 6 Mar 2021

5. Yang X, Yu Y, Xu J et al (2020) Clinical course and outcomes of critically ill patients with SARS-CoV-2 pneumonia in Wuhan, China: a single-centred, retrospective, observational study. Lancet Respir Med. https://doi.org/10.1016/S2213-2600(20):30079

6. Zhou F, Yu T, Du R et al (2020) Clinical course and risk factors for mortality of adult inpatients with COVID-19 in Wuhan, China: a retrospective cohort study. Lancet. https://doi.org/10. 1016/S0140-6736(20)30566-3

7. Richardson S, Hirsch JS, Narasimhan M et al (2020) Presenting characteristics, comorbidities, and outcomes among 5700 patients hospitalized with COVID-19 in the New York City Area. JAMA. https://doi.org/10.1001/jama.2020.6775

8. Battle D, Soler MJ, Sparks MA et al (2020) Acute kidney injury in COVID-19: emerging evidence of a distinct pathophysiology. JASN. https://doi.org/10.1681/ASN.2020040419

9. Wang D, Hu B, Hu C et al (2020) Clinical characteristics of 138 hospitalized patients with 2019 novel coronavirus-infected pneumonia in Wuhan, China. JAMA 323:1061-1069

10. Diao B, Wang $C$, Wang $R$, et al. Human kidney is a target for novel severe acute respiratory syndrome coronavirus 2 (SARS-CoV-2) infection. Preprint. medRxiv. https://doi.org/10.1101/2020.03.04. 20031120. Accessed 19 Apr 2020
11. Cheng Y, Luo R, Wang K et al (2020) Kidney disease is associated with inhospital death of patients with COVID-19. Kidney Int 97:829-838

12. Chu KH, Tsang WK, Tang CS et al (2005) Acute renal impairment in coronavirus-associated severe acute respiratory syndrome. Kidney Int 67(2):698-705

13. Ng JJ, Luo Y, Phua K, Choong AM (2020) Acute kidney injury in hospitalized patients with coronavirus disease 2019 (COVID-19): a meta-analysis. J Infect 81(4):647-679. https://doi.org/10.1016/j. jinf.2020.05.009

14. Hirsch JS, Ng JH, Ross DW, Northwell COVID-19 Research Consortium et al (2020) Acute kidney injury in patients hospitalized with COVID-19. Kidney Int. https://doi.org/10.1016/j.kint.2020. 05.006 (Online ahead of print. PMID: 32416116)

15. Guan W-J, Ni Z-Y, Hu Y et al (2020) Clinical characteristics of coronavirus disease 2019 in China. N Engl J Med 382:1708-1720

16. Huang C, Wang Y, Li X et al (2020) Clinical features of patients infected with 2019 novel coronavirus in Wuhan, China. Lancet 395:497-506

17. Kidney Disease: Improving Global Outcomes (KDIGO) Acute Kidney Injury Work Group (2012) KDIGO clinical practice guideline for acute kidney injury. Kidney Int 2:1-138

18. Chen N, Zhou M, Dong X et al (2020) Epidemiological and clinical characteristics of 99 cases of 2019 novel coronavirus pneumonia in Wuhan, China: a descriptive study. Lancet 395:507-513

19 Ye M, Wysocki J, William J, Soler MJ, Cokic I, Batlle D (2006) Glomerular localization and expression of angiotensin-converting enzyme 2 and angiotensin-converting enzyme: implications for albuminuria in diabetes. J Am Soc Nephrol 17:3067-3075

20. Batlle D, Soler MJ, Sparks MA, COVID-19 and ACE2 in Cardiovascular, Lung, and Kidney Working Group et al (2020) Acute kidney injury in COVID-19: emerging evidence of a distinct pathophysiology. J Am Soc Nephrol. https://doi.org/10.1681/ ASN.2020040419 (Online ahead of print. PMID: 32366514)

21 Yang HS, Wan C, Yi L et al (2020) Renal histopathological analysis of 26 postmortem findings of patients with COVID-19 in China. Kidney Int. https://doi.org/10.1016/j.kint.2020.04.003 (Published online ahead of print Apr 9, 2020)

22. Post A, den Deurwaarder ESG, Bakker SJL et al (2020) Kidney infarction in patients With COVID-19. Am J Kidney Dis. https:// doi.org/10.1053/j.ajkd.2020.05.004 (Online ahead of print. PMID: 32479921)

23. Tang N, Li D, Wang X, Sun Z (2020) Abnormal coagulation parameters are associated with poor prognosis in patients with novel coronavirus pneumonia. J Thromb Haemost 18:844-847

24. Arentz M, Yim E, Klaff L et al (2020) Characteristics and outcomes of 21 critically ill patients with COVID-19 in Washington state. JAMA 323:1612-1614

25. Wang L, Li X, Chen $\mathrm{H}$ et al (2020) Coronavirus disease 19 infection does not result in acute kidney injury: an analysis of 116 hospitalized patients from Wuhan, China. Am J Nephrol. https:// doi.org/10.1159/000507471

26. Chan L, Chaudhary K, Saha A et al (2021) AKI in hospitalized patients with COVID-19. J Am Soc Nephrol 32(1):151-160. https://doi.org/10.1681/ASN.2020050615 (Epub 2020 Sep 3. PMID: 32883700 doi: 10.1101/2020.08.16.20175992; posted on August 18, 202026)

27. Caplin NJ, Zhdanova O, Tandon M, et al. (2020) Acute peritoneal dialysis during the COVID-19 pandemic at Bellevue Hospital in New York City. medRxiv. Preprint. https://doi.org/10.1101/2020. 08.16.20175992. Accessed 18 Aug 2020

Publisher's Note Springer Nature remains neutral with regard to jurisdictional claims in published maps and institutional affiliations. 